\title{
High stability gel electrolytes for long life lithium ion solid state supercapacitor
}

\author{
Zhi Chen ${ }^{1,2, *}$, Junxiang $\mathrm{Li}^{1}$, Mengqiang $\mathrm{Wu}^{1}$, Jiaxuan Liao ${ }^{1,2}$ \\ ${ }^{1}$ School of Materials and Energy, University of Electronic Science and Technology of China, Chengdu 611731, People's Republic of \\ China \\ ${ }^{2}$ Yangtze Delta Region Institute (Quzhou), University of Electronic Science and Technology of China, Quzhou, Zhejiang 324000, China
}

\begin{abstract}
Lithium ion capacitors with liquid electrolyte are prone to leakage, combustion, explosion and other dangerous accidents. To solve these problems, the solid gel separator prepared by polyvinylidene fluoride - six fluoropropene (PVDF-HFP) is used in this work to improve the safety and stability of lithium ion supercapacitors. The PVDF-HFP based gel separator was used to replace the commercial separator and electrolyte in the lithium ion capacitor. The solid-state lithium ion supercapacitor was matched with porous carbon (PC) and hard carbon (HC). The maximum energy density of the device is $148.76 \mathrm{wh} / \mathrm{kg}$, even at the power density of $33.6 \mathrm{~kW} / \mathrm{kg}$, which still retains $20.6 \mathrm{wh} / \mathrm{kg}$. In addition, $83.3 \%$ capacity of solid-state lithium-ion supercapacitor is retained after 8000 times of charge and discharge. The requirements of high power energy density, high cycle stability and high safety are realized.
\end{abstract}

\section{INTRODUCTION}

Traditional energy storage devices (capacitors, batteries) generally use liquid electrolyte, which may cause unknown hazards in the process of charging and discharging. The solid electrolyte with high safety has been widely concerned by many researchers. Due to its stable structure, wide electrochemical window and heat resistance, and its ability to effectively prevent the generation of lithium dendrites, solid electrolytes are more suitable for fields requiring high safety and stability [1,2]. If solid electrolyte is used in lithium-ion capacitor, three-high energy storage devices with high power energy density, high cycle stability and high safety will be obtained. However, the current research focuses on liquid state and solid-state lithium-ion batteries, the research on solid-state lithium-ion capacitors is still less [3-6]. However, due to the special structure of lithium-ion capacitors, solid-state lithiumion capacitors can be studied by referring to solid-state lithium-ion batteries.

According to the composition of solid electrolyte membrane, it can be divided into organic polymer, inorganic solid and composite membrane $[7,8]$. In this work, PVDF-HFP solid polymer electrolyte membrane was studied. Among them, due to the existence of HFP, the copolymerization weakens the crystallinity of the membrane, making the interface between the electrode material and the electrolyte more stable $[9,10]$. In addition, in order to obtain a more porous membrane, the electrospinning process was used to prepare the membrane [11]. Finally, the PVDF-HFP solid-state separator with high porosity was prepared, which was used as a substitute for the commercial separator and electrolyte, and assembled with PC and $\mathrm{HC}$ to form a solid-state lithium-ion capacitor with high power energy density, high cycling and high safety. The maximum energy density of the device is $148.76 \mathrm{wh} / \mathrm{kg}$, even at the power density of $33.6 \mathrm{~kW} / \mathrm{kg}$, which still retains 20.6 wh $/ \mathrm{kg}$. In addition, $83.3 \%$ capacity of solid-state lithiumion supercapacitor is retained after 8000 times of charge and discharge. This provides some new ideas for the development of the next generation of lithium ion supercapacitors.

\section{Experimental methods}

\subsection{Preparation of PVDF-HFP electrolytes}

Adding 1.239 g PVDF-HFP particles to $10 \mathrm{ml}$ DMF solution and stirring for 12 hours, a uniform half gel solution was obtained. After that, the solution was electrospun into a white flake polymer and dried in a vacuum oven at $75{ }^{\circ} \mathrm{C}$ for 24 hours. Finally, the tablet press is used to cut it into the required diaphragm. Before assembling the button cell, it is necessary to immerse the diaphragm in the electrolyte for half an hour, and obtain the gel solid electrolyte. The preparation process is shown in Figure 1.

\subsection{Characterization of materials}

FE-SEM (jsm-5900lv, jeol) was used to characterize the microstructure of the membrane. The porosity is calculated by formula of porosity $=\left(\mathrm{w}_{\mathrm{t}}-\mathrm{w}_{0}\right) / \mathrm{\rho V}$ (wt and

\footnotetext{
* Corresponding author: drzhichen@163.com
} 
$\mathrm{W}_{0}$ are the mass $(\mathrm{g})$ before and after the test, $\mathrm{V}$ is the volume $\left(\mathrm{cm}^{3}\right), \rho$ is the density of $\mathrm{n}$-butanol $\left.\left(\mathrm{g} / \mathrm{cm}^{3}\right)\right)$. The liquid absorption rate is calculated by formula of liquid absorption rate $=\left(\mathrm{w}_{\mathrm{t}}-\mathrm{w}_{0}\right) / \mathrm{w}^{0}$. Put the diaphragm directly above the alcohol lamp for combustion test to verify its thermal stability.

\subsection{Test of electrochemical performance}

The gel membrane (after immersion in electrolyte) and two stainless steel sheets were assembled into a battery for EIS test, and ionic conductivity was calculated according to formula of $\sigma=\mathrm{L} / \mathrm{RS}$ (Where $\mathrm{R}$ is the impedance value after fitting, $\mathrm{S}$ is the area of the gel membrane $\left(\mathrm{cm}^{2}\right)$, and $\mathrm{L}$ is the thickness of the gel membrane $(\mathrm{cm}))$. In addition, the button cell was assembled with stainless steel sheet and lithium sheet for electrochemical stability window test. $80 \mathrm{wt} \%$ active materials, $10 \mathrm{wt} \%$ PVDF and $10 \mathrm{wt} \%$ super $\mathrm{P}$ with proper amount of NMP were agitated and coated on the collector (anode copper foil, cathode aluminum foil), and then dried to obtain the electrode plate. In the glove box, electrode plates, lithium sheets, gel separators after absorbing the electrolyte (1 M LiPF6 in EC: DEC:DMC $(1: 1: 1))$ are assembled.

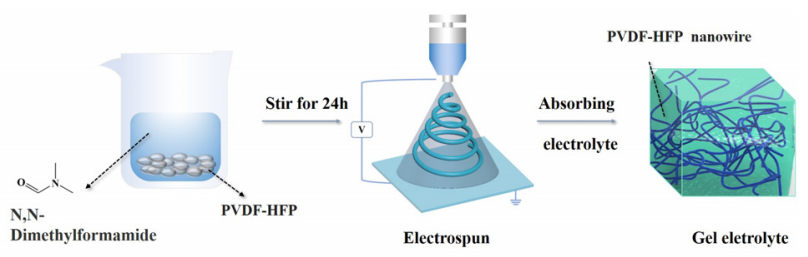

Figure 1 Preparation process of gel electrolytes.

\section{Results and discussion}

\subsection{Characterization of materials}

The SEM image of the gel electrolyte is shown in Figure 2a. After the treatment of electrospinning process, the microstructure shows a complex network structure of irregular filaments. The special structure leads to more pores and large specific surface area, which will promote the absorption of electrolyte and provide more $\mathrm{Li}+$ and PF6- ions channels, thus improving the electrochemical performance of the device. The absorbency and porosity are two key parameters for the gel membrane. High absorbency is conducive to promoting the migration rate of ions. High porosity can enhance the storage and absorption of electrolyte.

The photos and test results of the gel membrane before and after the absorption of electrolyte and glycol are shown in Figure 2b-e. It is seen that the liquid membrane shows the quasi solid morphology of the gel state after absorbing the liquid. The absorbency and porosity of gel electrolyte were $379.3 \%$ and $26.53 \%$ based on formula. Their good properties were caused by the porous structure resulting from electrospinning. Figure $2 \mathrm{~h}$ shows the combustion test of the gel membrane. It can be seen that the PVDF-HFP gel membrane obtained by electrospinning has very good heat resistance. During the test, the membrane does not burn, but it shrinks at the edge. Therefore, if there are accidents such as collision, overcharge and other easy to induce combustion, the gel diaphragm can provide higher security.

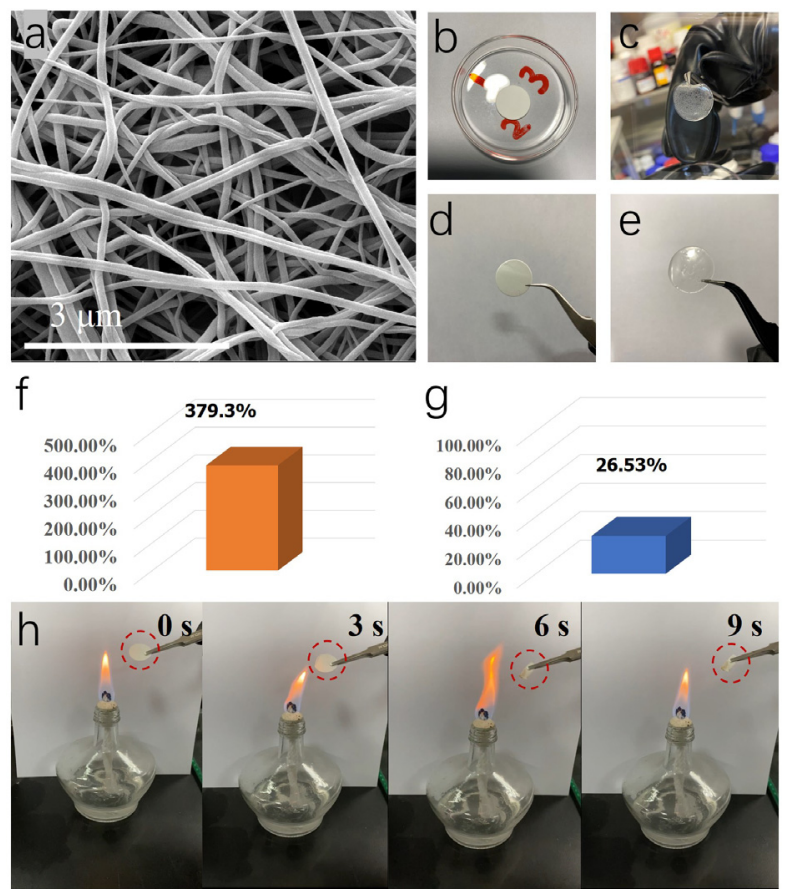

Figure 2. (a) SEM image of gel electrolyte. Before (b) and after (c) the absorption of electrolyte. Before (b) and after (c) the absorption of glycol. Porosity (f), liquid absorption rate (g) and combustion test of gel electrolyte.

\subsection{Electrochemical performance test}

Figure $3 \mathrm{a}$ is the EIS of the gel electrolyte. For the conventional test, the curve is composed of semicircle at high frequency and oblique line at low frequency. The semicircle is caused by the impedance caused by the movement of lithium ion and the reaction of electrode material. The two electrodes in the conductivity test of the gel membrane are stainless steel sheets, which do not react with the electrolyte, so that the diameter of the semicircle is infinitely enlarged, and the final curve is approximately a straight line. The conductivity of the gel membrane is $2.849 \mathrm{mS} / \mathrm{cm}$ by fitting the curve.

Electrochemical stability window is one of the key indexes to judge the electrochemical stability of membrane. In Figure $3 b$, the linear sweep voltammetric curve of the gel electrolyte and lithium sheet and stainless steel sheet are assembled. Between $2.5 \mathrm{~V}$ and $4.5 \mathrm{~V}$, the current of the battery maintains a relatively stable state. When it approaches $4.5 \mathrm{~V}$, its value rises rapidly, indicating that additional redox reaction occurs, which will destroy the structure of the membrane [106]. It should be noted that the test range of capacitive cathode is $2-4.5 \mathrm{~V}$, which may affect its electrochemical performance test, but battery anode $(0-3 \mathrm{~V})$ and lithium ion capacitor (1.5-4 V) are not affected by it. 

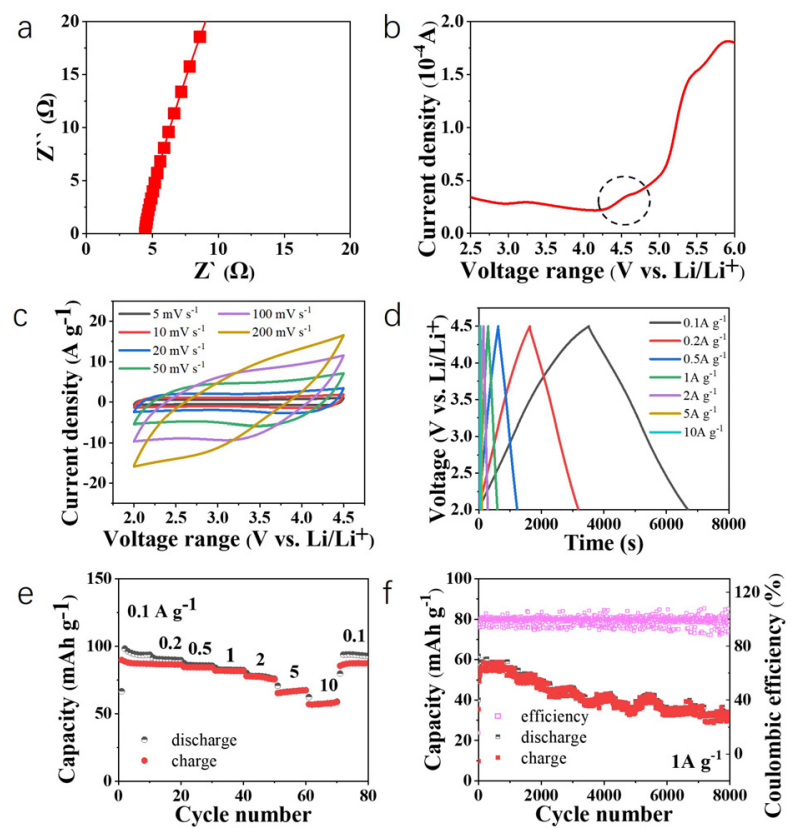

Figure 3. EIS (a) and LSV (b) of gel electrolyte. CV curve (c), charge-discharge curves (d), rate performance (e) and long cycle performance (f).

By setting the voltage range to $2.0-4.5 \mathrm{~V}$, the electrochemical performance of half cell was evaluated by using gel membrane as electrolyte. Figure $3 \mathrm{c}$ shows the $\mathrm{CV}$ curve at the scanning rate of $5-200 \mathrm{mV} / \mathrm{s}$. Even at $200 \mathrm{mV} / \mathrm{s}$, the curve is still quasi rectangular, indicating that cell has good reversibility. The charge-discharge curves at $0.1-10 \mathrm{~A} / \mathrm{g}$ are shown in Figure $4 \mathrm{~b}$, and the curves are basically quasi triangular, which can well show the ideal capacitance behavior. Figure $3 \mathrm{~d}$ shows the rate performance of $\mathrm{PC}$ at different current densities. In the electrochemical stability window test, it is found that the gel electrolyte may have additional reaction at about $4.5 \mathrm{~V}$, which may adversely affect the capacitive cathode test. The specific capacity of PC is $88.8 \mathrm{mAh} / \mathrm{g}$ at $0.1 \mathrm{~A} / \mathrm{g}$. When the current density up to $0.2,0.5,1,2$, 5 and $10 \mathrm{~A} / \mathrm{g}$, the specific capacity of $\mathrm{PC}$ is $86.8,84.5$, $82.0,77.6,66.7$ and $58.2 \mathrm{mAh} / \mathrm{g}$, respectively (Figure 3e). The specific capacity was maintained at $34.1 \mathrm{mAh} / \mathrm{g}$ after 8000 cycles of long cycle test at $5 \mathrm{~A} / \mathrm{g}$ (Figure $3 \mathrm{f}$ ). Although the electrochemical range of the gel diaphragm limits its electrochemical performance, it still performs better than other carbon materials, thanks to the excellent pore volume and super specific surface area as well as the pseudo capacitance effect caused by rich oxygen elements of PC.

By setting the voltage range to $0.01-3.0 \mathrm{~V}$, the electrochemical performance of $\mathrm{HC}$ was evaluated in the half cell with gel separator as electrolyte, as shown in Figure 4a. The CV curves and charge discharge curves are similar to those in liquid electrolyte. When the current density is $0.1,0.2,0.5,1,2,5 \mathrm{~A} / \mathrm{g}$, the specific capacity of the electrolyte is $250.3,216.4,190.9,171.8$, $152.8,119.5 \mathrm{mAh} / \mathrm{g}$, respectively. It is noteworthy that, thanks to the special structural stability of the gel electrolyte, which exhibited better results in the long cycle test at $1 \mathrm{~A} / \mathrm{g}$. This results implies that the solid lithium ion capacitor based on the gel electrolyte diaphragm will have better cycling stability.

A solid lithium ion capacitor was constructed by using PC as a capacitive cathode and using pre-lithium $\mathrm{HC}$ as battery anode and using PVDF-HFP gel membrane as electrolyte. Figure 5a shows the charge storage mechanism of solid state lithium-ion capacitors. In the charging process of $\mathrm{PC} / \mathrm{HC}, \mathrm{PF} 6-$ is absorbed by the porous structure and defects of PC cathode, while $\mathrm{Li}+$ is inserted into the interlayer space of $\mathrm{HC}$, and the reverse process occurs in discharge. In addition, choosing the working voltage range of 1.5-4 $\mathrm{V}$ can avoid the side reaction of gel septum during charging and discharging.
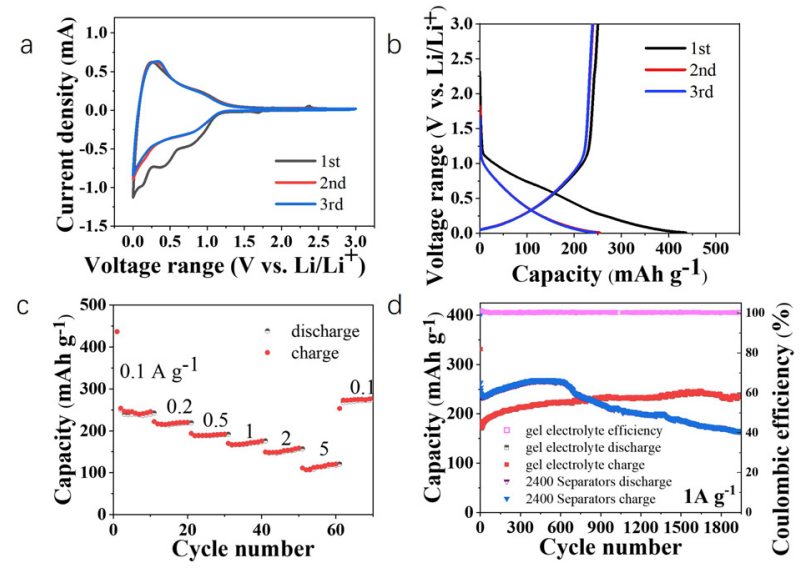

Figure 4. Electrochemical performance of $\mathrm{HC}$ based on gel electrolyte. CV curve(a), charge-discharge curve (b), rate performance (c) and long cycle performance (d) of HC.

The electrochemical performance test of $\mathrm{PC} / \mathrm{HC}$ solid state lithium ion capacitor is shown in figure $5 \mathrm{~b}$-e. Because cathode and anode have two different energy storage mechanisms, the shape of CV Curve is slightly different from the ideal situation. With the increase of scanning speed, the basic style of the curve is well preserved, there is no serious deformation, and it still shows an approximate symmetrical shape. The chargedischarge curves are triangular, which proves that it has excellent electrochemical reversibility. The power and energy density are calculated according to the chargedischarge curve. Its maximum energy density can reach $148.76 \mathrm{Wh} / \mathrm{kg}(100.36 \mathrm{~W} / \mathrm{kg})$, and it still retains 20.6 $\mathrm{Wh} / \mathrm{kg}$ at $33.6 \mathrm{~kW} / \mathrm{kg}$. The stability of the solid lithium ion capacitor has been greatly improved due to the better performance of the $\mathrm{HC}$ cell anode using the gel diaphragm. Under the high current density of $5 \mathrm{~A} / \mathrm{g}$, after 8000 charge discharge cycles, the energy density is stabilized to $83.3 \%$ of the initial capacity, and the decay rate of each cycle is only $0.00209 \%$. During the cycle, the internal resistance and lithium consumption may lead to the decrease of capacity. In short, a reasonable combination of anode and cathode and pre-lithium technology and the use of highly stable solid-state gel separator, $\mathrm{PC} / \mathrm{HC}$ solid state lithium ion capacitors show high energy power density, excellent cycle stability and good safety performance, the basic construction idea of "three-high" electrochemical energy storage equipment is realized. 
a

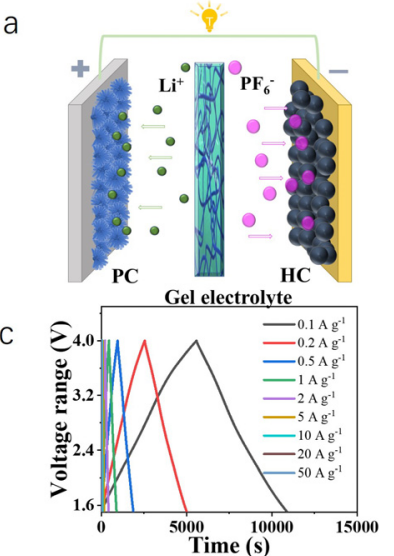

e

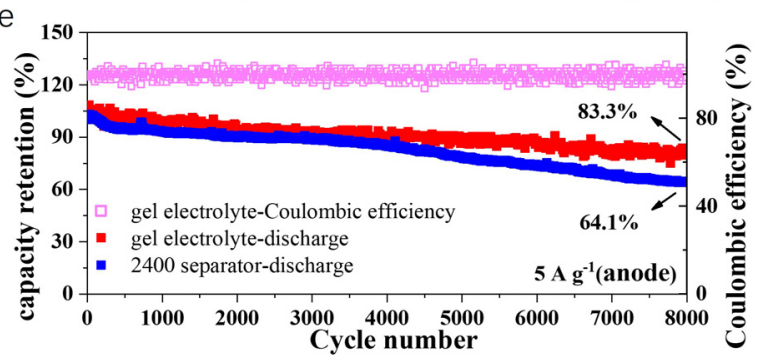

Figure 5. (a) Charge storage mechanism diagram of $\mathrm{PC} / / \mathrm{HC}$ capacitor. CV curve (a), charge-discharge curve (b), Ragone diagram (c) and long cycle performance (d) of $\mathrm{PC} / / \mathrm{HC}$ capacitor.

\section{Conclusion}

In summary, in order to solve the problem of safety hidden trouble in the traditional lithium ion capacitor, the PVDF-HFP based gel solid separator is used to replace the two parts of the separator and electrolyte in the lithium ion capacitor. The gel solid separator has high absorbency, good porosity and high heat resistance, and has excellent electrochemical properties. The gel solid electrolyte was assembled a full battery with high pore volume and large surface area PC as cathode and $\mathrm{HC}$ as anode. The obtained solid lithium ion capacitor device exhibits a high energy density of $148.76 \mathrm{Wh} / \mathrm{kg}$. Even at $33.6 \mathrm{~kW} / \mathrm{Kg}$, the energy density of $20.6 \mathrm{Wh} / \mathrm{kg}$ can be retained, comparable to that based on liquid electrolyte. In addition, the long cycle stability of the gel separator is better than that of the liquid lithium ion capacitor. After 8000 cycles of charging and discharging, the solid lithium ion capacitor keeps $83.3 \%$ capacity. So far, the solid-state lithium-ion capacitor $\mathrm{PC} / / \mathrm{HC}$ developed in this work provides a new generation of "three-high" energy storage equipment with high power and energy density, high cycle stability and high safety.

\section{Acknowledgements}

The authors gratefully acknowledge the support by Sichuan Science and Technology Program of 2018GZ0029, 2019YFH0002, 2020YFH0213, 2020YFG0357, 2020YFG0358, and 2019ZDZX0002, Science and Technology Commission Project of 18-163-
21-CH-002-003-01, and Central University Support plan of ZYGX2019Z022.

\section{References}

1. Z. Xu, M. Wu, Z. Chen, C. Chen, J. Yang, T. Feng, E. Paek and D. Mitlin, Adv. Sci. 6, 1802272 (2019)

2. C. Chen, M. Wu, Z. Xu, T. Feng, J. Yang, Z. Chen, S. Wang and Y. Wang, J Colloid Interf. Sci. 538, 267-276 (2019)

3. Z. Chen, Z. Xu, W. Li, C. Chen, J. Yang, J. Liu, F. Gong, J. Liao and M. Wu, ACS Appl. Energy Mater. 2, 5171-5182 (2019)

4. Z. Chen, J. Liao, W. Li, Y. Song, C. Chen, J. Yang, Z. Xu, T. Feng and M. Wu, Appl. Surf. Sci. 506, 144909 (2020)

5. Z. Chen, W. Li, J. Yang, J. Liao, C. Chen, Y. Song, S. A. Ali Shah, Z. Xu and M. Wu, J Electrochem. Soc. 167, 050506 (2020)

6. W. Li, Z. Chen, J. Hou, T. Xu, D. Liu, S. Leng, H. Guo, C. Chen, J. Yang and M. Wu, Appl. Surf. Sci. 515, 145902 (2020)

7. Q. Liu, Z. Geng, C. Han, Y. Fu, S. Li, Y.-b. He, F. Kang and B. Li, J Power Sources 389, 120-134 (2018)

8. A. M. Stephan, Eur. Polym. J 42, 21-42 (2006)

9. J. Jie, Y. Liu, L. Cong, B. Zhang, W. Lu, X. Zhang, J. Liu, H. Xie and L. Sun, J Energy Chem. 49, 80-88 (2020)

10. W. Liu, K. Wang, C. Li, X. Zhang, X. Sun, J. Han, X.-L. Wu, F. Li and Y. Ma, J Mater. Chem. A 6, 24979-24987 (2018)

11. J.-W. Jung, C.-L. Lee, S. Yu and I.-D. Kim, Journal of Mater. Chem. A 4, 703-750 (2016) 